\title{
Philadelphia-positive case negative for JAK2 V617F mutation with hyperdiploidic karyotype: A case report
}

\author{
DRAGOMIRA NIKOLOVA ${ }^{1,2}$, VERA DAMYANOVA ${ }^{1,2}$, VASIL HRISCHEV $^{2}$, MARIA MARKOVA $^{2}$, \\ LUBOMIR MITEV $^{3}$, ASELINA ASENOVA ${ }^{3}$, ATANAS RADINOV ${ }^{2}$ and DRAGA TONCHEVA ${ }^{1}$ \\ ${ }^{1}$ Department of Medical Genetics, Medical Faculty, Medical University Sofia; ${ }^{2}$ Clinic of Hematology, \\ University Hospital 'St. Ivan Rilski', 1431 Sofia; ${ }^{3}$ Department of Clinical Laboratory and Immunology, \\ Military Medical Academy, 1606 Sofia, Bulgaria
}

Received February 3, 2019; Accepted August 16, 2019

DOI: $10.3892 /$ mco.2019.1933

\begin{abstract}
Chronic myeloid leukemia (CML) is one of the most common hematological malignancies and accounts for 15-20\% of all leukemia cases. The cytogenetic marker of CML is the presence of Philadelphia chromosome $(\mathrm{Ph})$ in $>95 \%$ of patients. The current case reports a 83-year old woman who was directed to the genetic laboratory for a cytogenetic and molecular-genetic analysis suspected to be $\mathrm{Ph}$ positive $[(+)]$. Karyotype analysis of a bone marrow sample revealed a hyperdiploid karyotype in a part of $\mathrm{Ph}(+)$ cells with additional chromosomes 8, 10 and 12. Restriction analysis for V617F JAK2 mutation was negative, while the quantitative RT-qPCR assay indicated BCR-ABL/ABL transcript at the level of $120 \%$ International Scale $(I S)$. Generally cytogenetic complexities are important in the prognostic evaluation of CML. Besides the Ph chromosome, a variet of chromosomal aberrations may be associated with CML. A total of 5-10\% of these cases show complex translocations involving another chromosome. The current case is $\mathrm{Ph}(+)$ demonstrating an additional hyperdiploid karyotype clone with three additional autosomes $(8,10$ and 12). This case highlights the significance of cytogenetic abnormalities on the prognosis of CML.
\end{abstract}

\section{Introduction}

Chronic myeloid leukemia (CML) is one of the most common hematologic malignancies and accounts for $15 \%$ of all cases of leukemia in adults (1). The incidence of CML is approximately $1.6 / 100,000$. The cytogenetic marker of the disease is the presence of a distinctive Philadelphia chromosome $(\mathrm{Ph})$ in more than $95 \%$ of the patients (2). It is a reciprocal translo-

Correspondence to: Dr Dragomira Nikolova, Department of Medical Genetics, Medical Faculty, Medical University Sofia, 2 Zdrave Street, 1431 Sofia, Bulgaria

E-mail: nikolova_dragomira@yahoo.com;dmb@abv.bg

Key words: chronic myeloid leukaemia, Philadelphia chromosome, hyperdiploid karyotype cation between the long arms of chromosomes 9 and 22. The translocation involves the transfer of the Abelson or $A B L 1$ gene on chromosome 9 to the breakpoint cluster region, $B C R$, of chromosome 22, resulting in a fusion $B C R / A B L$ gene. The fusion gene produces $\mathrm{BCR} / \mathrm{ABL}$, a tyrosine kinase with deregulated activity that plays a key role in the development of CML.

Our case is an 83-year old woman who is directed to the genetic laboratory for a cytogenetic and molecular-genetic analysis suspected to be $\mathrm{Ph}$ positive [(+)]. Her initial diagnosis is primary aplastic anemia with additional diagnosis of primary (essential) hypertonia. The anamnesis is taken both from medical documentation and the statements of patient's relatives. She is accepted for the first time in the clinic having leukocytosis with neutrophilia, moderate-to-severe anemic syndrome-microcytic, hypochromic anemia; the thrombocytes are in the reference ranges. She has a consuming syndrome accompanied with a preserved and/or increased appetite and sore throat. Fever and feverish night sweats are lacking, there is no bleeding.

High levels of leucocytes have been diagnosed February, 2018. The leucocyte numbers increase in the following several months up to $85 \mathrm{G} / 1$. The levels from March, 2005 are documented as follows: St 12\%; Sg 17\%; Mo 2\%; Ly 10\%; Eo 5\%; $\mathrm{Bl}+$ ProM 25\%; M 21\%; Meta M 9\%.

Upon her acceptance in the hematological ward she is adequate and orientated. Her skin and mucosal membranes are pale pink with isolated suffusions and hematomas; no icterus is present. Peripheral lymph nodes are not enlarged on palpation; breathing is clear vesicular, double-sided, without wheezing. Cardiovascular system-there is arrhythmic cardiac activity, clear tones, systolic noise at Ao and cardiac apex. Stomach-soft, painless; liver-1-2 cm below the ribs' arch; spleen-non-enlarged. There are no swellings of the limbs.

\section{Materials and methods}

At the Clinic of Hematology the patient is subjected to routine diagnostic procedures: Whole blood count test and biochemical analysis (Table I), morphological analysis of the blood cells, restriction analysis for V617F in JAK2 gene (Fig. 1), quantitative molecular-biological analysis (Real-time PCR) at the time 
Table I. WBC and biochemical analysis result of the patient at the time of her acceptance in the ward.

\begin{tabular}{|c|c|c|c|c|c|c|c|}
\hline Biochemical characteristic & Result & Units & Reference values & Parameter & Result & Units & Referent values \\
\hline WBC & 69.77 & $\mathrm{x} 10^{9} / 1$ & $3.50-10.50$ & Gluc & 5.58 & $\mathrm{mmol} / \mathrm{l}$ & $3.50-6.10$ \\
\hline $\mathrm{RBC}$ & 4.34 & $\mathrm{x} 10^{12} / 1$ & $3.70-5.30$ & Creatinine & 161.00 & $\mu \mathrm{mol} / 1$ & up to 96.00 \\
\hline HGB & 87 & $\mathrm{~g} / 1$ & $120-160$ & TBil & 8.50 & $\mu \mathrm{mol} / 1$ & up to 21.00 \\
\hline HTC & 0.295 & $1 / 1$ & $0.360-0.480$ & ASAT & 27.60 & $\mathrm{U} / 1$ & up to 32.00 \\
\hline $\mathrm{MCV}$ & 67.8 & $\mathrm{fl}$ & $80.0-96.0$ & ALAT & 7.00 & $\mathrm{U} / 1$ & up to 33.00 \\
\hline $\mathrm{MCH}$ & 19.90 & $\mathrm{pg}$ & $27.0-33.0$ & $\mathrm{LDH}$ & 2783.00 & $\mathrm{U} / 1$ & up to 460 \\
\hline $\mathrm{MCHC}$ & 294 & $\mathrm{~g} / 1$ & $300-360$ & $\mathrm{Na}$ & 143.00 & $\mathrm{mmol} / \mathrm{l}$ & $135-151$ \\
\hline Platelets & 182 & $\mathrm{x} 10^{9} / 1$ & $130-360$ & $\mathrm{~K}$ & 3.20 & $\mathrm{mmol} / \mathrm{l}$ & $3.5-5.6$ \\
\hline$\%$ lymphocytes & 3.60 & $\%$ & $20.0-48.0$ & $\mathrm{Cl}$ & 103.00 & $\mathrm{mmol} / \mathrm{l}$ & $93-112$ \\
\hline$\%$ monocytes & 6.30 & $\%$ & $1.0-11.0$ & $\mathrm{Fe}$ & 15.80 & $\mu \mathrm{mol} / 1$ & $6.60-28.00$ \\
\hline$\%$ eosinophils & 0.80 & $\%$ & Up to 6.5 & TIBC & 50.30 & $\mu \mathrm{mol} / 1$ & $42.00-78.00$ \\
\hline$\%$ basophils & 7.40 & $\%$ & Up to 2.0 & UIBC & 34.50 & $\mu \mathrm{mol} / 1$ & $27.8-63.6$ \\
\hline$\%$ neutrophils & 88.00 & $\%$ & $40.0-70.0$ & CRP & 0.20 & $\mathrm{mg} / \mathrm{l}$ & $<6$ \\
\hline No lymphocytes & 2.54 & $\mathrm{x} 10^{9} / 1$ & $1.00-4.00$ & & & & \\
\hline No monocytes & 4.36 & $\mathrm{x} 10^{9} / 1$ & Up to 0.80 & & & & \\
\hline No eosinophils & 0.59 & $\mathrm{x} 10^{9} / 1$ & Up to 0.50 & & & & \\
\hline No basophils & 5.14 & $\mathrm{x} 10^{9} / 1$ & Up to 0.14 & & & & \\
\hline No neutrophils & 61.39 & $\mathrm{x} 10^{9} / 1$ & $2.00-7.00$ & & & & \\
\hline MPV & 9.40 & $\mathrm{fl}$ & $6.3-12.5$ & & & & \\
\hline
\end{tabular}

WBC, whole blood count; RBC, red blood cells; HGB, hemoglobin; HTC, hematocrit; MCV, mean corpuscular volume; MCH, mean corpuscular hemoglobin; MCHC, mean corpuscular hemoglobin concentration; MPV, mean platelet volume; Gluc, glucose; TBil, total bilirubin; ASAT, aspartate transaminase; ALAT, alanine transaminase; LDH, lactic acid dehydrogenase; Na, sodium; K, potassium; Fe, iron; TIBC, total iron binding capacity; UIBC, unsaturated iron binding capacity; CRP, C-reactive protein.

of her first visit and three months after treatment (Fig. 2), and karyotyping (Fig. 3). For all procedures and tests the patient has provided a written informed consent.

PCR amplification and restriction analysis for JAK2 V716F mutation detection. DNA is extracted from $200 \mu 1$ venous blood taken in $\mathrm{K}_{2}$ EDTA tube using QIAamp DNA Mini kit (Qiagen). JAK2 mutation has been checked as a part of the routine diagnostic procedure of the Clinic of Haematology when chronic myeloproliferative process is suspected. DNA is amplified using $J A K 2$ codon 617 mutation specific primers (V617F) (JAK2F 5'-GGGTTTCCTCAGAACGTT-3' and $J A K 2 R$ 5'-TCATTGCTTTCCTTTTTC-3') for 32 cycles using Taq polymerase (Qiagen), annealing temperature of $60^{\circ} \mathrm{C}$, and standard amplification conditions as described previously by Baxter et al (3). The amplified 460-bp fragment is enzymatically digested using BsaXI restriction enzyme (BioLabs ${ }^{\mathrm{TM}}$ ). Fragments of three different sizes (241, 189 and $30 \mathrm{bp}$ ) are received after digestion of the wild type allele, while the mutant allele remains undigested (Fig. 1). Digested fragments are separated in $2 \%$ agarose gel. Visualization of the restriction fragments is achieved by ethidium bromide (Fig. 1).

$R T$-qPCR analysis. It is carried out using the Cepheid GeneXpert ${ }^{\circledR}$ platform. RNA has been automatically extracted from $4 \mathrm{ml}$ venous blood and converted to cDNA. Xpert BCR-ABL kit is used for quantitative detection of BCR-ABL chromosomal translocation mRNA transcripts and the ABL

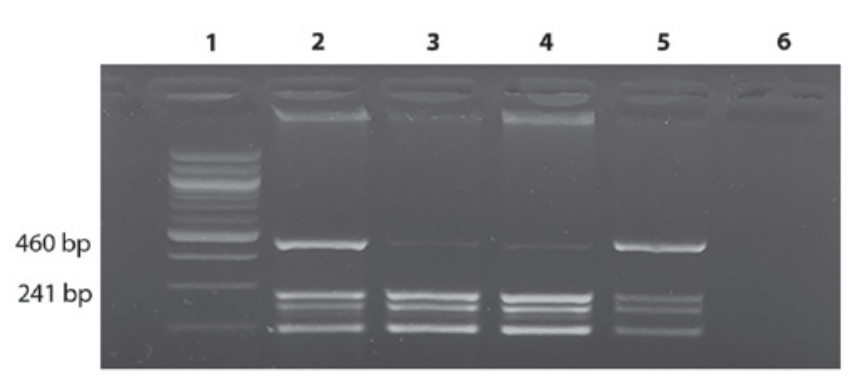

Figure 1. Restriction analysis following reverse transcription-quantitative PCR of JAK2 exon 12 gene. BsaXI endonuclease is used to specifically cut the PCR product on $2 \%$ agarose gel. Lane 1 indicates 100 bp ladder, lane 2 and 5 indicate individuals, heterozygote for JAK2 V617F mutation, lane 3 and 4 indicates individuals homozygous by the normal allele (lane 3 corresponds to the patient from the presented case) and lane 6 indicates the negative control.

endogenous control mRNA transcripts in peripheral blood specimen from patients with CML. The Xpert BCR-ABL Ultra quantifies the BCR-ABL mRNA level on the $I S$ and is calibrated to the first World Health Organization (WHO) international genetic reference panel for quantitation of BCR-ABL mRNA. The GeneXpert software calculates the $\% \mathrm{BCR}-\mathrm{ABL} / \mathrm{ABL}(I S)$ using the following equation where the Delta $\mathrm{Ct}(\Delta \mathrm{Ct})$ value is obtained from $\mathrm{ABL} C \mathrm{Ct}$ minus BCR-ABL Ct: \%BCR-ABL/ABL $I S=E_{\Delta C t}{ }^{(\Delta \mathrm{Ct})}$ x 100 x Scaling Factor (SF) (4). The efficiency value is embedded in the barcode of the Xpert BCR-ABL Ultra cartridge; the SF is lot-specific (Xpert ${ }^{\circledR}$ BCR-ABL Ultra Handbook) (Fig. 2). 
A

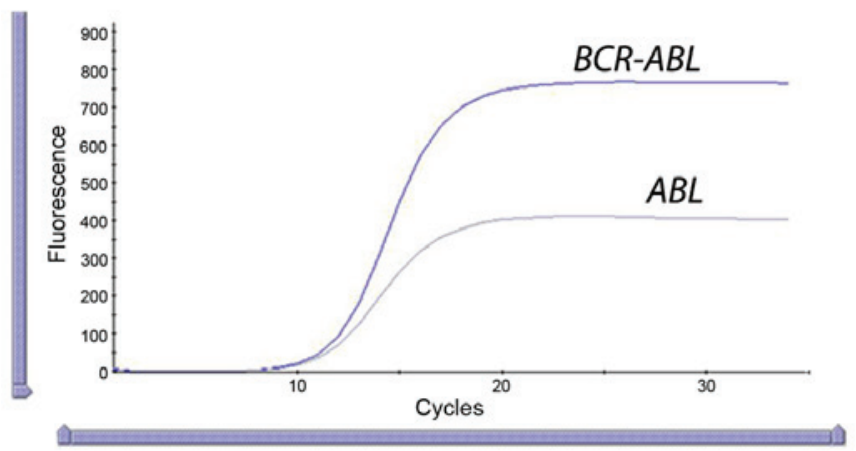

B

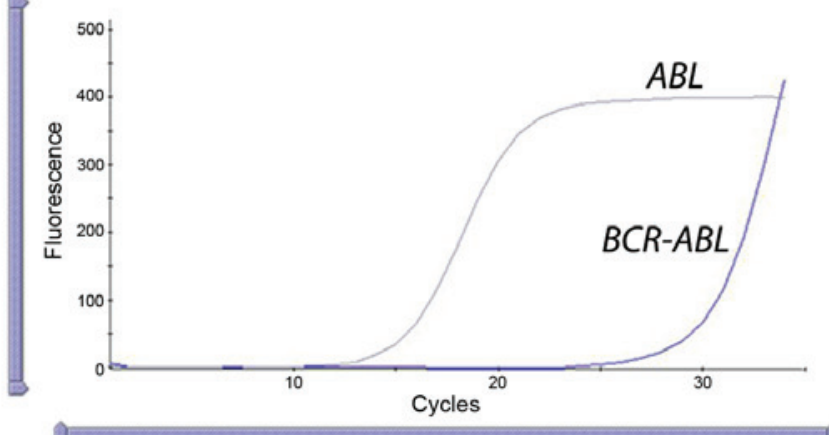

Figure 2. Cepheid ${ }^{\circ}$ RT-q PCR analysis of the patient showing a positive amplification curve (A) Result from the RT-q PCR analysis at the time of diagnosis (B) Result from the RT-q PCR after three months of Tasigna treatment. Primary curve of the patient showing amplification of ABL (internal control) and the BCR-ABL gene is represented in both cases. RT-q, reverse transcription-quantitative; BCR, breakpoint cluster region; ABL, Abelson.

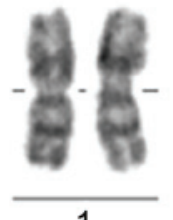

1

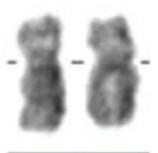

6

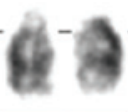

13

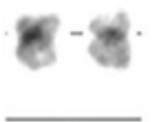

19

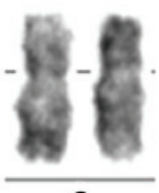

2

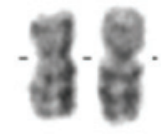

7

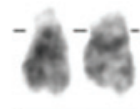

14

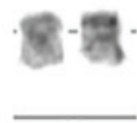

20

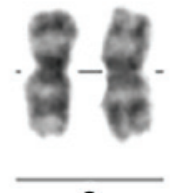

3

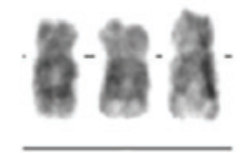

8
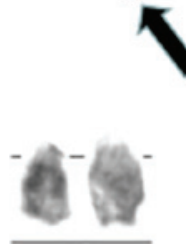

15

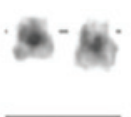

21

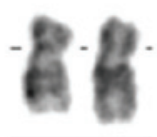

9

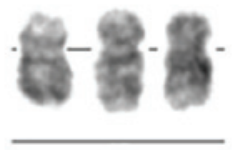

10

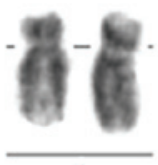

4

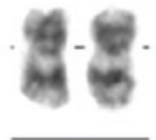

11

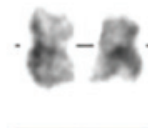

16

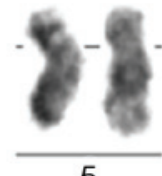

5

Figure 3. Chromosome banding analysis indicating a complex hyperdiploid karyotype with numerical aberration and a total number of 49 chromosomes $(49, \mathrm{XX},+8,+10,+12)$. Additional autosomes are indicated by black arrows while Philadelphia chromosome is encircled and indicated by a red arrow.

Cytogenetic analysis. The bone marrow sample of the patient is taken by trepanobiopsy and sent to the cytogenetic laboratory of the Military Medical Academy (Sofia, Bulgaria). The cells are cultured in RPMI culture medium supplied with antibiotics and growth factors at $37^{\circ} \mathrm{C}$ for $24 \mathrm{~h} .70 \mu \mathrm{l}$ of colcemid is added to the sample and incubated in $37^{\circ} \mathrm{C}$ for $20 \mathrm{~min}$. After centrifugation at $1,500 \mathrm{rpm}, 0.075 \mathrm{M} \mathrm{KCl}$ is added and the sample is incubated at $37^{\circ} \mathrm{C}$ for $10 \mathrm{~min}$. After two times fixation and centrifugation, several microscopic slides are prepared. They undergo standard Giemsa staining and are analyzed under microscope (Fig. 3).

\section{Results}

The morphological analysis of cells' populations shows hypercellular bone marrow with an altered ratio between the neutrophil, granulocyte and erythroblast clones up to 12.1:1 (granulocytes 0.90; erythroblasts 0.07 ); hyperplasia of the neutrophil population with sustained maturation, increase of myelocytes and promyelocytes. Index of maturation 1.3 (reference values 0.5-0.8). Cytological features of gigantism in a part of the metamyelocytes. Substantially reduced erythroblast population with single representatives of all 
maturation forms. Well-presented megakaryocyte apparatus with a dominating population of polyploidic granulated megakaryocytes. The conclusion of the morphological analysis is 'Chronic myeloproliferative process'. The ratio of different cell populations in the bone marrow is as follows: $1.5 \%$ lymphocytes, $87.9 \%$ granulocytes, $0.1 \%$ myeloblasts, $6.2 \%$ mature monocytes, $4.2 \%$ promonocytes. The whole blood count test of the patient and the biochemical analysis result at the time of her acceptance in the haematological ward of 'St. Ivan Rilski' Hospital is presented in Table I.

Based on the myelography the patient is directed for molecular-biological analysis for JAK2 mutation and $\mathrm{Ph}$ chromosome. The result of the restriction analysis is presented in Fig. 1 showing the genotype status of the patient (homozygous carrier of two wild type alleles for V617F JAK2 gene mutation).

Quantitative Real-time PCR analysis of the patient is performed (May, 2018) to confirm the presence of the molecular marker of CML-fusion gene $B C R / A B L$. The graph clearly shows the positive result of the amplification for both $A B L$ (endogenous control) and $B C R-A B L$ genes. The software estimates automatically the level of the fusion gene of $120 \%$ $(I S)$ on the International Standardized Scale (Fig. 2A). Real-time analysis is performed second time (September, 2018) after four months' treatment with Tasigna (Nilotinitib) to monitor the molecular improvement of the patient. The result demonstrates $0,0019 \%$ (IS) (Fig. 2B) level which is much less compared to the patient's earlier data.

Karyotyping is performed after 24-h cultivation time of stimulated bone marrow specimen. Chromosomes are obtained, stained following Giemsa standard protocol and subsequently analyzed under light microscope (5). The cytogenetic analysis shows two different clones of cells: Hyperdiploid with additional chromosomes 8, 10 and 12 and $\mathrm{Ph}$ chromosome; and second clone which is $\mathrm{Ph}(+)$ with no hyperdiploidy (Fig. 3).

\section{Discussion}

Hyperdiploidy is a phenomenon of having additional chromosomes rather than the diploid chromosome number in the karyotype. According to Onodera et al, hyperdiploid karyotype arises by a simultaneous gain of multiple chromosomes from a diploid karyotype during a single abnormal cell division (6). Generally hyperdiploidy is not a common event in CML patients (7). Other chromosome changes, such as chromosomal translocations, are more common, especially at the time of blast transformation (8). Hyperdiploidy could be a bad prognostic factor in CML $(7,9)$. Further investigation on the topic shows that the existing statements about hyperdiploidy are ambiguous.

A case report (10) presents a $\mathrm{Ph}(+) \mathrm{CML}$ case with hyperdiploidic karyotype and an additional T315I kinase domain (KD) mutation in the $B C R-A B L$ gene. The patient responded well after therapy with Nilotinib. Though T315I mutation remained after the treatment, targeted drug eliminated the hyperdiploid clone (10).

In a study on acute lymphoblastic leukemia (ALL) in children high hyperdiploidy is characterized by a favourable prognosis (11). A paper studying chromosomal aberrations in pediatric and adult ALL defines high hyperdiploidy as present in $25-30 \%$ of children with favorable prognosis and $7-8 \%$ in adults with favorable/intermediate prognostic value (12). In multiple myeloma patients hypodiploidy but not hyperdiploidy, is a poor indicator for the disease's development (13). According to some authors (14-16) hypodiploid DNA content is related to a poor response to chemotherapy and a very short survival time; according to others (17) hyperdiploid karyotype is associated with a better prognosis. The chromosomes which usually appear in addition in ALL karyotypes are 21, X, 14, $6,18,4,17$ and 10 which tend to be gained in blasts (18). The acquisition of chromosome 21 is seen in $95 \%$ of hyperdiploid cases.

There are only few reports about hyperdiploid karyotypes in CML cases. Rojas et al analyzes 63 cases with CML and not even one additional chromosome is found (19). There is only one case with i(17q); and one case with 21q deletion (19). Roland and Blahey reported a case of a patient with breast cancer who developed hyperdiploidy at the accelerated phase of the disease, near blast crisis, presenting with seven additional chromosomes besides $\mathrm{Ph}$ chromosome $(+6,+8,+11,+18,+19$, $+20,+21)(8)$. Gains of chromosomes 6 and 19 are common in CML hyperdiploic karyotypes (8). Other commonly reported chromosomes are 8 and $19 \mathrm{in} \mathrm{Ph}(+)$ cases of CML (20). In a study of 256 patients with CML only one is with 51 chromosomes and trisomy $+6,+10,+13$ and +19 (21). Chromosomes $+7,+8,+9,+10,+12,+15,+19$ are reported in a case with near triploid karyotype in pre-imatinib mesylate CML patient with T315I mutation in BCR-ABL kinase domain (10).

The reported case in this study has been diagnosed as CML as for of an absence of a blast infiltration in the bone marrow. The myelogram and the immunophenotyping characteristics of the cells reveal the percentage of myeloblasts in the bone marrow estimated at $0.1 \%$. Besides being $\mathrm{Ph}(+)$, the patient shows a hyperdiploid karyotype with additional chromosomes 8,10 and 12 (see 'Results'). To our knowledge, this is the only report of chromosome 12 in a hyperdiploid CML patient.

The underlying mechanism of hyperdiploidy and how this phenomenon can potentially influence the expression profile of the genes is still unknown (22). The influence of hyperdiploidy in the course of CML remains a disputable question in literature. From clinical point of view, the chromosomal gain is associated with increased risk for blast crisis $(23,24)$. To our knowledge, there is only one more report which presents higher percentage level of BCR-ABL transcript detected quantitatively and expressed in \% (IS) (25). The detected level of the BCR-ABL transcript was measured to be $187 \%$ $(I S)$ in bone marrow and $152 \%$ (IS) in peripheral blood of a 28-year-old patient.

As a whole the cytogenetic complexities play major role in the prognostic evaluation of CML. Along with the Ph chromosome, various chromosomal aberrations can be associated with CML. $5-10 \%$ of these cases showing complex translocation involving another chromosome in addition to the $\mathrm{Ph}$ chromosome. Our case is $\mathrm{Ph}(+)$ with an additional hyperdiploid clone with trisomy $8,10,12$. Cases like this could sometimes present with higher results of $\mathrm{BCR}-\mathrm{ABL} / \mathrm{ABL}$ transcripts detected quantitatively.

\section{Acknowledgements}

Not applicable. 


\section{Funding}

No funding was received.

\section{Availability of data and materials}

All data generated or analyzed during the present case study are included in this article.

\section{Authors' contributions}

DN performed the restriction analysis, Real-time PCR test, performed the literature review and wrote the manuscript, VD assisted in the laboratory data interpretation, VH and MM interpreted the clinical data of the patient and monitored the treatment, LM and AA performed the cytogenetic analysis, AR and DT acquired relevant data and revised the manuscript critically. All authors have read and approved the manuscript.

\section{Ethics approval and consent to participate}

Not applicable.

\section{Patient consent for publication}

Written informed consent was obtained from the patient for the publication of the obtained data.

\section{Competing interests}

The authors declare that they have no competing interests.

\section{References}

1. Tamascar I and Ramanarayanan J: Targeted treatment of chronic myeloid leukemia: Role of imatinib. Onco Targets Ther 2: 63-71, 2009.

2. Jabbour E and Kantarjian H: Chronic myeloid leukemia: 2016 update on diagnosis, therapy, and monitoring. Am J Hematol 91: 252-265, 2016.

3. Baxter EJ, Scott LM, Campbell PJ, East C, Fourouclas N, Swanton S, Vassiliou GS, Bench AJ, Boyd EM, Curtin N, et al: Acquired mutation of the tyrosine kinase JAK2 in human myeloproliferative disorders. Lancet 365: 1054-1061, 2005.

4. Livak KJ and Schmittgen TD: Analysis of relative gene expression data using real-time quantitative PCR and the 2(-Delta Delta C(T)) method. Methods 25: 402-408, 2001.

5. Safaei A, Shokripour M and Omidifar N: Bone marrow and karyotype findings of patients with pancytopenia in southern iran. Iran J Med Sci 39: 333-340, 2014.

6. Onodera N, McCabe NR and Rubin CM. Rubin: Formation of a hyperdiploid karyotype in childhood acute lymphoblastic leukemia. Blood 80: 203-208, 1992.

7. Belurkar S, Manohar C and Kurien A: Chronic myeloid leukemia with hyperdiploidy: A case report with review of literature. Indian J Med Sci 67: 188-192, 2013.

8. Roland B and Blahey WB: A case of near-triploidy in chronic myelogenous leukemia. Cancer Genet Cytogenet 121: 96-98, 2000 .
9. Yehuda O, Abeliovich D, Ben-Neriah S, Sverdlin I, Cohen R, Varadi G, Orr R, Ashkenazi YJ, Heyd J, Lugassy G and Ben Yehuda D: Clinical implications of fluorescence in situ hybridization analysis in 13 chronic myeloid leukemia cases: $\mathrm{Ph}$-negative and variant Ph-positive. Cancer Genet Cytogenet 114: 100-107, 1999.

10. Al-Achkar W, Moassass F, Ikhtiar A, Liehr T, Othman MA and Wafa A: Hyperdiploidy associated with T315I mutation in BCR-ABL kinase domain in an accelerated phase-chronic myeloid leukemia case. Mol Cytogenet 7: 89, 2014.

11. Paulsson K and Johansson B: High hyperdiploid childhood acute lymphoblastic leukemia. Genes Chromosomes Cancer 48: 637-660, 2009

12. Mrózek K, Harper DP and Aplan PD: Cytogenetics and molecular genetics of acute lymphoblastic leukemia. Hematol Oncol Clin North Am 23: 991-1010, v, 2009.

13. Smadja NV, Bastard C, Brigaudeau C, Leroux D and Fruchart C; Groupe Français de Cytogénétique Hématologique: Hypodiploidy is a major prognostic factor in multiple myeloma. Blood 98: 2229-2238, 2001.

14. Barlogie B, Alexanian R, Dixon D, Smith L, Smallwood L and Delasalle K: Prognostic implications of tumor cell DNA and RNA content in multiple myeloma. Blood 66: 338-341, 1985.

15. Latreille J, Barlogie B, Dosik G, Johnston DA, Drewinko B and Alexanian R: Cellular DNA content as a marker of human multiple myeloma. Blood 55: 403-408, 1980.

16. Morgan RJ Jr, Gonchoroff NJ, Katzmann JA, Witzig TE, Kyle RA and Greipp PR: Detection of hypodiploidy using multi-parameter flow cytometric analysis: A prognostic indicator in multiple myeloma. Am J Hematol 30: 195-200, 1989.

17. Garcia-Sanz R, Orfão A, González M, Moro MJ, Hernández JM, Ortega F, Borrego D, Carnero M, Casanova F, Jiménez R, et al: Prognostic implications of DNA aneuploidy in 156 untreated multiple myeloma patients. Castelano-Leones (Spain) cooperative group for the study of monoclonal gammopathies. Br J Haematol 90: 106-112, 1995.

18. Heerema NA, Raimondi SC, Anderson JR, Biegel J, Camitta BM, Cooley LD, Gaynon PS, Hirsch B, Magenis RE, McGavran L, et al: Specific extra chromosomes occur in a modal number dependent pattern in pediatric acute lymphoblastic leukemia. Genes Chromosomes Cancer 46: 684-693, 2007.

19. Rojas A, Pineda L, González S, Soto M, Avila E, Urdaneta B, Prieto-Carrasquero $\mathrm{M}$ and González R: Chromosomal abnormalities in malignant hematologic diseases. Acta Cient Venez 51: 109-114, 2000 (In Spanish).

20. Werner M, Kaloutsi V, Buhr T, Delventhal S, Vykoupil KF and Georgii A: Cytogenetics of chronic myelogenous leukemia (CML) correlated to the histopathology of bone marrow biopsies. Ann Hematol 63: 201-205, 1991.

21. Meng CY: Cytogenetics and molecular studies in chronic myeloid leukemia. Clements University, 2001.

22. Stagno F, Vigneri P, Consoli ML, Cupri A, Stella S, Tambè L, Massimino M, Manzella L and Di Raimondo F: Hyperdiploidy associated with a high BCR-ABL transcript level may identify patients at risk of progression in chronic myeloid leukemia. Acta Haematol 127: 7-9, 2012.

23. AM B: Cancer cytogenetics. The principles of clinical cytogenetics. Totowa, New Jersey, Humana Press Inc, 1999.

24. Godley LA and Le Beau MM: Williams Hematology. 8 edition. Cytogenetics and molecular abnormalities. McGraw Hill, 2008.

25. Wang Z, Zen W, Meng F, Xin X, Luo L, Sun H, Zhou J and Huang L: Chronic myeloid leukemia with variation of translocation at $(\mathrm{Ph})$ [ins $(22 ; 9)(\mathrm{q} 11 ; \mathrm{q} 21 \mathrm{q} 34)]$ : A case report. Int J Clin Exp Pathol 8: 13707-13710, 2015. 